\title{
Correction to: Inward international students in China and their contributions to global common goods
}

\section{Lin $\operatorname{Tian}^{1} \cdot$ Nian Cai Liu ${ }^{1}$}

Published online: 7 November 2020

(c) Springer Nature B.V. 2020

\section{Correction to: Higher Education https://doi.org/10.1007/s10734-020-00522-5}

The original version of this article unfortunately contained a mistake. The presentation of Table 7 was incorrect. The correct Table 7 is now shown here.

The original article has been corrected.

Table 7 A framework for inward student mobility from a common goods view

\begin{tabular}{llll}
\hline Sets & Dimensions & Keywords \\
\hline Set 1 & Common & Individual & Benefit \\
Set 2 & Governmental & Institutional & Influence \\
Set 3 & National & Global & Balance \\
\hline
\end{tabular}

Source: Summarized by the authors.

The original article can be found online athttps://doi.org/10.1007/s10734-020-00522-5

Nian Cai Liu

ncliu@sjtu.edu.cn

1 Shanghai Jiao Tong University, Room 241, Chen Ruiqiu Building, 800 Dongchuan Road. Minhang District, Shanghai 200240, China 